\title{
Independência Funcional entre Comportamentos de Falante e Ouvinte Relativos a Paisagens Desconhecidas
}

\author{
Sarah Moraes de Simões ${ }^{1}$ \\ ${ }^{1}$ Instituto Brasiliense de Análise do Comportamento, \\ DF, Brasil. \\ Carlos Augusto de Medeiros ${ }^{2}$ \\ ${ }^{2}$ Centro Universitário de Brasília, DF, Brasil. \\ Eduardo Walcacer Viegas ${ }^{2}$ \\ ${ }^{2}$ Centro Universitário de Brasília, DF, Brasil.
}

\begin{abstract}
Resumo: Este estudo investigou o efeito de treinos sucessivos na emissão não treinada de comportamentos de ouvinte e falante após o treino do outro operante. Doze adultos universitários típicos foram divididos em dois grupos com base na ordem de exposição aos treinos e testes em três fases experimentais. Os participantes do Grupo Ouvinte-Falante passaram por treinos de ouvinte e testes de falante, e os demais foram submetidos a treinos e testes na ordem inversa. Os cinco estímulos utilizados por fase foram palavras inventadas e paisagens desconhecidas por eles. Os participantes do Grupo Falante-Ouvinte atingiram o critério de dependência funcional nas três fases experimentais. No outro grupo, apenas um integrante apresentou dependência funcional na primeira fase experimental, sendo observada melhora gradual no desempenho nos testes ao longo do experimento para os demais. Discutiu-se a eficácia dos treinos sucessivos na ocorrência de dependência funcional condicionalmente a aspectos do procedimento.
\end{abstract}

Palavras-chave: Comportamento Verbal, Dependência Funcional, Adultos, Treinos Sucessivos.

\section{Functional Independence Between Speaker-Listener Behaviors Related to Unknown Landscapes}

\begin{abstract}
This study investigated the effect of successive training on the untrained emission of listener and speaker behaviors after the training of the other operant. Twelve typical undergraduates were divided into two groups based on order of training and testing in three experimental phases. The participants of the Listening-Speaker Group underwent listener training and speaker test; and the other participants were subjected to training and tests in the reverse order. The five stimuli used per phase were invented words and unknown landscapes. The participants of the Speaker-Listener Group reached the criterion of functional dependence in the three experimental phases. In the other group, only one participant presented functional dependence in the first experimental phase, with a gradual improvement in performance in the tests throughout the experiment for the remaining subjects. The efficacy of successive training in the occurrence of functional dependence associated to aspects of the procedure was discussed.
\end{abstract}

Keywords: Verbal Behavior, Functional Dependence, Adults, Successive Training Sessions. 


\title{
Independencia Funcional entre Comportamientos de Hablante y Oyente Relacionados a Paisajes Desconocidos
}

\begin{abstract}
Resumen: El presente estudio investigó el efecto de los entrenamientos sucesivos en la emisión no entrenada de las conductas del oyente y el hablante después del entrenamiento del otro operante. Se dividieron doce universitarios adultos típicos en dos grupos según el orden de entrenamiento y los test en tres fases experimentales. Los participantes del Grupo Oyente-Hablante pasaron por entrenamientos de oyentes y test de los hablantes; y los otros participantes se sometieron a entrenamientos y test en el orden inverso. Los cinco estímulos utilizados por fase fueron palabras inventadas y paisajes desconocidos por ellos. Los participantes del Grupo Hablante-Oyente alcanzaron el criterio de dependencia funcional en las tres fases. En el otro grupo, solo un participante presentó dependencia funcional en la primera fase, con una mejora gradual en el rendimiento en los test a lo largo del experimento para los demás. Se discutió la eficacia de los entrenamientos sucesivos en la aparición de dependencia funcional condicionalmente a aspectos del procedimiento.
\end{abstract}

Palabras clave: Comportamiento Verbal, Dependencia Funcional, Adultos, Sesiones de Entrenamiento Sucessivas.

No livro Verbal behavior, Skinner (1957) introduz o conceito de comportamento verbal referindo-se a fenômenos humanos que anteriormente eram tratados sob o rótulo da linguagem. De acordo com Skinner, o comportamento verbal é um tipo de operante, assim, é controlado por suas consequências, seu contexto de ocorrência e suas variáveis motivacionais (e.g., estados de privação e estimulação aversiva). A principal diferença entre o comportamento verbal e os demais operantes é que, no primeiro, as consequências são mediadas por outras pessoas. Skinner dá o nome de ouvinte para a pessoa que media as consequências ao comportamento verbal do falante, ou seja, aquele que emite a resposta verbal.

O comportamento do ouvinte, de acordo com Skinner, é controlado por estímulos discriminativos verbais emitidos pelo falante, podendo ser verbal ou não (Skinner, 1957; 1989/1991). Já o comportamento do falante é aquele que produz efeitos apenas no ambiente social, podendo ser categorizado em diferentes tipos, a partir de sua topografia (falada ou escrita), de seus antecedentes (verbais ou não verbais) e de suas consequências (generalizadas ou específicas).

A partir das definições de comportamento de falante e ouvinte, observa-se que estes são controlados por variáveis diferentes. Desse modo, uma das conclusões de Skinner é que, se uma pessoa aprende a emitir comportamentos de ouvinte sob controle discriminativo de certos estímulos verbais (e.g., uma palavra), não necessariamente ela emitirá comportamentos de falante que produzam os mesmos estímulos verbais sem um treino específico. Também não se deve pressupor que, quando um comportamento verbal com dada topografia de respostas (e.g., uma palavra) é treinado, ocorra, sem treinos específicos, a emissão de comportamento de ouvinte sob o controle discriminativo de estímulos verbais advindos da mesma resposta. Para Skinner (1957), portanto, os comportamentos de ouvinte e falante são independentes funcionalmente. Porém, a partir das interações com a comunidade verbal, esses comportamentos podem se tornar funcionalmente dependentes, o que é corriqueiro em adultos típicos (Skinner, 1957).

Com o objetivo de verificar empiricamente as digressões teóricas de Skinner acerca da independência funcional entre comportamentos de ouvinte e falante, diversos estudos foram conduzidos (Cuvo \& Riva, 1980; Germano \& Medeiros, 2020, Guess, 1969; Guess \& Baer, 1973; Lee, 1981; Lemgruber, 2013; Medeiros, Germano, \& Gonçalves, 2016; Viegas \& Medeiros, 2019; Wynn \& Smith, 2003). Estes estudos, em sua maioria, buscaram investigar também que condições de treino favoreciam a transposição entre 
os operantes, ou seja, a emissão não treinada de um dos comportamentos a partir do treino do outro.

Outra teoria sobre linguagem na perspectiva analítico-comportamental foi proposta por Horne e Lowe (1996). Em suma, a teoria foi criada para tentar explicar o caráter generativo da linguagem. Desde então, pesquisas empíricas e teóricas foram conduzidas para investigar esse fenômeno (Bao, Sweatt, Lechago, \& Antal 2017; Fiorile \& Greer, 2007; Gilic \& Greer, 2011; Greer \& Longano, 2010; Greer \& Speckman, 2009; Greer, Stolfi, Chavez-Brown, \& Rivera-Valdes, 2005) utilizando um procedimento de instrução de múltiplos exemplares (MEI). Mais recentemente, autores como Miguel (2016) e Hawkins, Gautreaux e Chiesa (2018), em uma tentativa de sistematizar estudos sobre o caráter generativo da linguagem, independentemente do arcabouço teórico comportamental, nomearam os diferentes procedimentos para facilitar a compreensão da comunidade científica. Hawkins et al. (2018) nomearam estudos que envolvem o treino de ouvinte/falante e teste do operante oposto como nomeação bidirecional.

Um dos primeiros estudos a investigar a dependência funcional entre os comportamentos de ouvinte e falante foi realizado por Guess (1969). Nessa pesquisa, os participantes com desenvolvimento atípico foram ensinados a apontar para objetos que apareciam em pares ou em uma unidade após a verbalização do nome do objeto (i.e. treino de ouvinte) pelo experimentador no plural e no singular, respectivamente. Após esse treino, os participantes foram solicitados a nomear esses objetos apresentados em pares ou unidades. Os resultados indicaram a independência funcional entre os comportamentos. Guess e Baer (1973) ampliaram essa linha de investigação adicionando treinos e testes na ordem inversa, isto é, treinos de falante e testes de ouvinte. Participaram do estudo quatro novos participantes, duas crianças e dois adultos com desenvolvimento atípico. Metade dos participantes passou por treinos de ouvinte e testes de falante, enquanto os demais foram treinados e testados na função oposta. Com exceção de um participante treinado como ouvinte e testado como falante, os demais apresentaram independência funcional entre os comportamentos de ouvinte e falante.

Cuvo e Riva (1980) investigaram se os resultados reportados por Guess (1969) e Guess e Baer (1973) eram devidos ao atraso no desenvolvimento dos participantes. Desse estudo, participaram dez crianças com desenvolvimento típico e dez crianças com desenvolvimento atípico. O comportamento de ouvinte consistiu em o participante apontar para uma moeda após o experimentador emitir seu nome, e o comportamento de falante, em nomear as moedas apresentadas pelo experimentador. Como em Guess e Baer (1973), metade dos participantes foi exposta à ordem de treino e teste, ouvinte-falante, e a outra metade foi exposta à ordem inversa, falante-ouvinte. Contrariando os dados de Guess (1969) e Guess e Baer (1973), os resultados apontaram para a dependência funcional principalmente no sentido falante-ouvinte. Os autores discutem que não houve diferença qualitativa entre aquisição e generalização dos repertórios entre os participantes, ressaltando apenas uma diferença quantitativa, visto que os participantes com desenvolvimento atípico necessitaram de maiores exposições aos treinos para atingirem os critérios de submissão aos testes.

Lee (1981) também trabalhou com os comportamentos de ouvinte e falante, todavia os estímulos a serem tateados nos treinos e testes de falante eram as posições (esquerda e direita, na frente e atrás, em cima e embaixo) relativas de pares de estímulos. Participaram dos três experimentos crianças típicas e atípicas, sendo comparada, novamente, a ordem de treino e teste (falante-ouvinte ou ouvinte-falante). Além disso, após treinos e testes dos comportamentos de ouvinte e falante, Lee (1981) invertia os significados nos nomes das posições para verificar se o treino prévio dos comportamentos de ouvinte e falante favoreceria a emissão não treinada de um dos operantes após o treino do outro (i.e., treinos sucessivos). Tal procedimento não resultou em melhora no desempenho. Assim como Cuvo e Riva (1980), a ordem falante-ouvinte produziu mais ocorrências de dependência funcional, a qual se mostrou inexistente na ordem ouvinte-falante.

De maneira geral, os estudos de Guess (1969), Guess e Baer (1973), Cuvo e Riva (1980) e Lee (1981) apontam que os repertórios de ouvinte e falante são independentes funcionalmente, ao menos no que se refere à ordem ouvinte-falante, tanto em pessoas com desenvolvimento típico como atípico. Com base nos pressupostos teóricos realizados por Skinner (1957), em conjunto com os dados empíricos, um impacto foi causado na comunidade cientifica comportamental no sentido de direcionar os cientistas para a criação de formas mais efetivas de programas 
de ensino de linguagem, principalmente para pessoas com atraso na linguagem. Inicialmente, com base nos estudos feitos até então, Lovaas (2003) havia proposto que seria melhor ensinar comportamentos de ouvinte antes dos de falante. Contudo, levando em consideração estudos mais recentes, Petursdottir e Carr (2011) e Contreras, Cooper e Kahng (2019) argumentaram justamente o contrário, isto é, que seria mais efetivo realizar treinos de falante e testes de ouvinte. Um estudo que demonstra tal fato foi realizado por Pérez-Gonzáles, Cereijo-Blanco e Carnerero (2014). Em um delineamento intrassujeito, avaliou-se se seriam mais eficientes para a emissão de comportamentos novos os treinos de ouvinte ou de falante. Nesse procedimento, utilizaram-se dois tipos de estímulo e duas formas de realizar o treino de ouvinte. De maneira geral, esse estudo corroborou os anteriores por ter sido observada maior emissão de comportamentos novos no sentido falante-ouvinte. No entanto eles também observaram a emissão de comportamentos novos na ordem inversa, e verificou-se diferença no desempenho a depender do procedimento e estímulo envolvido.

Com o objetivo de ampliar esses estudos, que foram realizados exclusivamente com crianças e pessoas com desenvolvimento atípico, Medeiros et al. (2016) resolveram investigar se os mesmos resultados seriam replicados em adultos plenamente inseridos em uma comunidade verbal. Para Skinner (1957), mesmo em adultos verbalmente habilidosos, é comum notar instâncias em que comportamentos de ouvinte e falante são independentes. Por exemplo, muitos falantes nativos do português conseguem ler em inglês com proficiência, ao passo que demonstram muita dificuldade na escrita. Ao mesmo tempo, outros nativos podem conseguir emitir várias palavras e sentenças em inglês como falantes, mas não ser capazes de respondê-las como ouvintes quando tentam assistir a um filme em inglês sem legenda.

Para investigar a dependência funcional em adultos, Medeiros et al. (2016) realizaram dois experimentos envolvendo comportamentos de falante e de ouvinte com universitários. No Experimento 1, metade dos participantes foi submetida à ordem falante-ouvinte (Grupo Falante-Ouvinte) e a outra metade, à ordem inversa (Grupo Ouvinte-Falante). Os estímulos/topografias de respostas verbais utilizados nas três fases foram palavras trissílabas inventadas, sendo cinco palavras diferentes por fase.
Nas duas primeiras fases, utilizaram-se figuras de objetos conhecidos (óculos, copo d'água, carro etc.) e, na última fase, caracteres desconhecidos.

No treino de ouvinte, diante da emissão de uma das palavras, os participantes tinham de apontar para um dentre cinco cartões possíveis (figuras conhecidas nas Fases 1 e 2 e caracteres desconhecidos na Fase 3). Nos treinos, os acertos e erros foram consequenciados, enquanto nos testes não houve consequências programadas para as respostas dos participantes. Nos treinos e testes de falante nas Fases 1 e 2, os participantes tinham de emitir o nome referente a um dos cartões diante de uma sentença emitida pelo experimentador com suposta função de operação estabelecedora condicionada. Apresentava-se a sentença no intuito de estabelecer a função reforçadora condicionada dos cartões numa tentativa de manipulação de variáveis motivacionais em adultos. Por exemplo, para a figura do copo d'água, foi criada a sentença "Você está com sede" como forma de aumentar o valor reforçador de receber a figura com copo d'água e, assim, evocar a resposta SIDEPO, que era a topografia de respostas referentes a essa figura. Em outros estudos acerca de independência funcional, como os de HalleSundberg (1987) e Nuzzolo-GomezeGreer (2004), que envolveram participantes com desenvolvimento atípico, as operações estabelecedoras puderam ser manipuladas diretamente. Já na terceira fase, o que estabelecia o valor reforçador dos caracteres desconhecidos eram molduras em formas abstratas que, para que fossem completas, o participante deveria emitir a palavra relativa ao caractere dentro da forma que se encaixaria em cada moldura.

Os resultados replicaram os demais estudos com crianças e participantes típicos, ou seja, dependência na ordem falante-ouvinte e independência na ordem ouvinte-falante. Houve uma queda no desempenho na terceira fase do Grupo falante-ouvinte, porque, segundo os autores, nos treinos de falante, o comportamento do participante poderia ter ficado sob o controle da forma da moldura, e não sob o controle do ideograma japonês. Assim, nos testes de ouvinte, como as molduras não eram apresentadas, o ideograma não exerceria controle sobre o responder discriminativo. Devido ao bom desempenho dos participantes que passaram pelo treino de falante primeiro nas Fases 1 e 2, não foi possível observar os efeitos de treinos sucessivos. 
Com relação aos testes de falante após treinos de ouvinte, os autores apontam que predominou independência funcional. Uma possibilidade de explicação para o fraco efeito dos treinos sucessivos nesses participantes, segundo os autores, pode ter sido o número reduzido de fases. Para testar essa possibilidade, o Experimento 2 de Medeiros et al. (2016) replicou o primeiro, adicionando mais três fases para cada um dos grupos de novos participantes. Foi incluída mais uma fase com objetos conhecidos e mais duas com objetos desconhecidos. Novamente, observou-se dependência funcional para os participantes do Grupo Falante-Ouvinte, com manutenção do alto desempenho mesmo nas fases com formas desconhecidas. Os autores atribuem esse resultado à retirada dos caracteres desconhecidos, de modo que as discriminações entre os estímulos se deram apenas com base nas formas das molduras. Já para o Grupo Ouvinte-Falante foi possível observar melhora no desempenho ao longo das fases para a maior parte dos participantes, o que sugeriu efeito dos treinos sucessivos.

$\mathrm{O}$ uso das sentenças nos dois experimentos de Medeiros et al. (2016) é questionável como operação estabelecedora. Como se tratavam de estímulos facilmente nomeáveis, é muito provável que cadeias intraverbais tenham sido incidentalmente estabelecidas nos treinos e testes (Horne \& Lowe, 1996), o que pode ter favorecido ou prejudicado a emissão dos operantes de ouvinte e falante não treinados. $\mathrm{O}$ uso de figuras desconhecidas na Fase 3 do segundo experimento tinha o objetivo de evitar o controle intraverbal, porém resultou em redundância no controle de estímulos, o que pode ter ocasionado a queda no desempenho. Na realidade, esse é um dos desafios na realização de pesquisa sobre independência funcional em adultos, ou seja, eliminar o efeito de variáveis pré-experimentais que podem interferir nos resultados.

Com vistas a evitar o efeito da história de condicionamento com os estímulos envolvidos de modo a viabilizar pesquisas com adultos acerca de independência funcional, este estudo replicou o Experimento 1 de Medeiros et al. (2016) com o uso de fotos de paisagens reais desconhecidas aos participantes. Utilizando-se esse material, objetivou-se minimizar o controle intraverbal induzido pelas figuras facilmente nomeáveis de Medeiros et al. (2016).

\section{Método}

\section{Participantes}

Participaram da pesquisa doze universitários, com média de idade de trinta anos, ingênuos em relação ao procedimento. Eles foram convidados a participar da pesquisa no campus da faculdade. A disponibilidade de tempo para estar presente nas sessões experimentais foi o critério de escolha dos participantes. Todos aceitaram participar voluntariamente da pesquisa assinando o termo de consentimento livre e esclarecido (TCLE). O projeto de pesquisa foi aprovado pelo comitê de ética do Centro Universitário de Brasília (UniCEUB), sob número de processo CAAE 02465512.7.0000.0023.

\section{Local}

A pesquisa foi realizada em salas de estudos de um centro universitário de Brasília, com aproximadamente três metros quadrados. A sala era provida de atenuação de ruídos nas paredes, iluminação no teto e um ar-condicionado no canto superior. As mobílias eram apenas uma mesa redonda, na qual foi realizado o experimento, e duas cadeiras, sendo uma para o experimentador e outra para o participante.

\section{Materiais}

Foram utilizados quinze cartões com imagens de paisagens desconhecidas pelos participantes, sendo que cinco delas eram referentes a cidades, cinco a fazendas e as demais, a paisagens de praias. Também foram utilizados protocolos de registro que continham os blocos de tentativa de cada treino e teste.

\section{Estímulos}

Nessa pesquisa, foram utilizadas quinze palavras trissílabas inventadas ao acaso sem significado para os participantes. Em cada fase experimental, havia cinco palavras cuja função era de estímulo discriminativo ou de topografia de resposta, a depender do comportamento treinado-testado. Foram selecionadas quinze imagens de paisagens desconhecidas para a execução desse procedimento. Essas imagens eram paisagens de cidades, fazendas ou praias, a depender da fase experimental. As imagens eram fotos reais ou, ainda, desenhos retirados de animações e jogos. $\mathrm{O}$ Quadro 1 apresenta as imagens e as palavras às quais elas se referem. 


\section{Quadro 1}

Figuras de paisagens, sentenças e palavras utilizadas para as três fases experimentais

\begin{tabular}{|c|c|c|c|c|c|}
\hline \multicolumn{2}{|c|}{ Fase 1} & \multicolumn{2}{|c|}{ Fase 2} & \multicolumn{2}{|c|}{ Fase 3} \\
\hline Figura & Palavra/Sentença & Figura & Palavra/Sentença & Figura & Palavra/Sentença \\
\hline inter & $\begin{array}{l}\text { PELOMI/Você } \\
\text { acaba de comprar } \\
\text { um barco e quer } \\
\text { aproveitar o sol } \\
\text { com os amigos. }\end{array}$ & & $\begin{array}{l}\text { ROFIMA/Ao } \\
\text { acordar você gosta } \\
\text { de tomar leite } \\
\text { tirado na hora. }\end{array}$ & & $\begin{array}{l}\text { GARILO/Você } \\
\text { não vê a hora de } \\
\text { entrar de férias e } \\
\text { aproveitar a sombra } \\
\text { e água fresca. }\end{array}$ \\
\hline & $\begin{array}{l}\text { MATROLI/Você } \\
\text { não pode sair de } \\
\text { casa sem protetor } \\
\text { solar e garrafinha } \\
\text { de água. }\end{array}$ & & $\begin{array}{l}\text { PAZONO/Nos } \\
\text { finais de semana } \\
\text { você costuma } \\
\text { cavalgar. }\end{array}$ & & $\begin{array}{l}\text { LEBORI/Você } \\
\text { adora mergulhar } \\
\text { com os peixes em } \\
\text { águas claras. }\end{array}$ \\
\hline & $\begin{array}{l}\text { RUDIPE/Você tem } \\
\text { visão empresarial } \\
\text { e deseja fazer } \\
\text { negócios. }\end{array}$ & & $\begin{array}{l}\text { DEMIRA/Está na } \\
\text { época de preparar } \\
\text { a terra para o } \\
\text { plantio. }\end{array}$ & & $\begin{array}{l}\text { COPESU/Está na } \\
\text { hora de estrear sua } \\
\text { prancha nova. }\end{array}$ \\
\hline & $\begin{array}{l}\text { SIMOFA/Você não } \\
\text { gosta de calmaria } \\
\text { nem de se sentir só. }\end{array}$ & & $\begin{array}{l}\text { BIVUTA/Você vai } \\
\text { passar o final de } \\
\text { semana com } \\
\text { boa música. }\end{array}$ & & $\begin{array}{l}\text { PULINU/ Neste } \\
\text { calor não há nada } \\
\text { melhor que } \\
\text { uma água de } \\
\text { coco gelada. }\end{array}$ \\
\hline & $\begin{array}{l}\text { ZAMOTA/Você } \\
\text { não tem carro } \\
\text { e, no dia a dia, } \\
\text { utiliza transporte } \\
\text { coletivo. }\end{array}$ & & $\begin{array}{l}\text { SUNEJA/Você vai } \\
\text { passar o feriado } \\
\text { com os amigos, e a } \\
\text { casa vai ficar cheia. }\end{array}$ & & $\begin{array}{l}\text { ZILINA/ Às vezes, } \\
\text { é preciso ficar só e } \\
\text { incomunicável. }\end{array}$ \\
\hline
\end{tabular}

\section{Procedimento}

Os participantes foram distribuídos de maneira aleatória em dois grupos de tamanhos iguais: Grupo Ouvinte e Grupo Falante. Ambos os grupos passaram por três fases experimentais que variaram apenas nas imagens utilizadas. A única diferença entre eles foi em relação aos comportamentos treinados e testados. O Grupo Ouvinte, em cada fase experimental, passou pelo treino de ouvinte, teste de falante, treino de falante, teste de manutenção de ouvinte e, a um segundo, teste de falante. O Grupo Falante foi submetido a treinos e testes com as funções inversas das do Grupo Ouvinte.

Inicialmente, antes dos procedimentos da primeira fase experimental, realizou-se um treino ecoico com os participantes com todas as palavras do experimento. O objetivo foi verificar se eles conseguiriam pronunciar todas as palavras utilizadas no estudo, excluindo, dessa forma, a possibilidade do baixo rendimento do participante nos testes devido à dificuldade em emitir as palavras do estudo. Cada palavra deveria ser pronunciada de forma correta duas vezes em até 45 tentativas. Caso esse critério não fosse alcançado, o participante seria excluído do experimento e outro seria chamado em seu lugar. No treino ecoico, o experimentador emitia cada palavra do estudo, uma por vez, e solicitava que o participante a repetisse. Em caso de acerto, o experimentador dizia "correto" e indicava uma nova palavra. Em caso de erro, o experimentador dizia "tente novamente" e pronunciava a palavra mais uma vez para o participante ecoar. Uma nova palavra só era apresentada caso o participante conseguisse ecoar a anterior.

Nos treinos de ouvinte, o experimentador posicionava as cinco imagens de estímulos referentes à fase experimental sobre a mesa. Com as imagens na mesa, 0 experimentador solicitava que o participante pegasse a imagem referente ao nome emitido pelo experimentador. 
Por exemplo, caso o experimentador dissesse: "pegue para mim BUMICA", e o participante pegasse a imagem correspondente a "BUMICA", era considerado acerto. Caso o participante pegasse a imagem correta, seu comportamento era consequenciado com verbalizações do experimentador como "parabéns" ou "muito bem”. Se o participante pegasse uma imagem incorreta, o experimentador sinalizava o erro e, em seguida, pedia novamente que ele pegasse o cartão correspondente: "Não, [nome do participante], este não é BUMICA. Pegue para mim BUMICA". O experimentador só apresentava uma nova palavra após o participante emitir a resposta correta. Os treinos de ouvinte foram organizados em blocos de dez tentativas em que cada estímulo apareceria duas vezes, de modo que ordem de apresentação era semirrandômica, o que se repetiu em todos os treinos e testes. O critério de encerramento do treino de ouvinte foi de acertar um bloco sem cometer erros. Caso o critério não fosse atingido em dez blocos, o participante era dispensado do experimento mediante agradecimento por sua participação.

Nos testes de ouvinte, o procedimento utilizado foi similar ao do treino. As diferenças foram na forma como as respostas do participante foram consequenciadas e em sua duração. Nesse procedimento, tanto erros quanto acertos não foram consequenciados, cabendo ao experimentador apenas registrar em seu protocolo de registros o desempenho do participante. A duração do teste de ouvinte foi de um bloco com dez tentativas, sendo que cada estímulo era testado duas vezes. O critério para os comportamentos de falante e ouvinte serem considerados dependentes funcionalmente foi acertar ao menos oito tentativas, ou seja, $80 \%$ de acertos.

No treino de falante, as imagens ficavam à disposição do experimentador. Foram criadas sentenças com função estabelecedora condicionada da mesma forma que ocorreu no estudo de Medeiros et al. (2016). Para cada imagem, criou-se previamente uma sentença com o objetivo de estabelecer um valor reforçador para a imagem. Cada sentença estabelecia o valor reforçador de um dos elementos presente em apenas uma das figuras do conjunto por fase. As sentenças utilizadas foram mostradas no Quadro 1. Após a apresentação da sentença, o experimentador perguntava: "Me diga: para onde você quer que eu te leve?".

Um exemplo do treino do repertório de falante seria o experimentador emitir a sentença: “Ao acordar você gosta de tomar leite tirado na hora. Me diga: para onde você quer que eu te leve?". Caso o participante emitisse a resposta correta, que, no caso, seria "ROFIMA", correspondente à imagem da fazenda que tinha uma vaca, $o$ experimentador sinalizaria o acerto falando "parabéns", "muito bem" e entregaria o cartão referente a essa topografia de palavra. Nas situações em que o participante emitia o nome de um estímulo presente no experimento, mas se referia a outra sentença, o experimentador entregaria o cartão solicitado pelo participante e só então sinalizaria o erro. Então o experimentador corrigiria o participante emitindo a topografia da palavra que seria considerada correta. Só seria iniciada uma nova tentativa com uma nova sentença após o participante emitir a resposta referente àquela sentença. Caso ele emitisse uma palavra não existente no experimento, o experimentador sinalizaria o erro e faria o mesmo procedimento corretivo descrito acima. $\mathrm{O}$ treino de falante foi organizado em blocos de dez tentativas, em que cada estímulo apareceria duas vezes. O critério de encerramento foi acertar um bloco sem cometer nenhum erro. Caso o critério não fosse atingido em dez blocos, o participante era dispensado do experimento mediante agradecimento por sua participação.

Os testes de falante ocorriam do mesmo modo que os treinos de falante, mas sem a apresentação de consequências programadas às tentativas dos participantes $\mathrm{e}$ ocorriam em um bloco de dez tentativas, de modo que cada estímulo-sentença era apresentado duas vezes.

\section{Resultados}

Não houve erros no treino ecoico. De acordo com o Gráfico 1, que mostra a porcentagem de tentativas corretas em todos os treinos e testes para os participantes do Grupo Ouvinte-Falante, é possível observar um aumento da porcentagem de acertos nos treinos de ouvinte ao se comparar a terceira fase experimental com a primeira. Além disso, é visível que, de maneira geral, todos os participantes precisaram de menos blocos de ouvinte para serem submetidos ao teste de falante na última fase. Também se pode observar que foram necessários menos blocos de treino de falante para atingir o critério de submissão aos testes do que treinos de ouvinte, com exceção de Marina (os nomes dos participantes apresentados aqui são fictícios para manter seu anonimato) nas três fases e de Lucas na primeira fase.

Com relação ao desempenho nos testes de falante, Júlia foi a única que obteve mais de $80 \%$ de acertos em todas as fases (Gráfico 1), sendo também a única a 
apresentar mais de $80 \%$ de acertos no primeiro teste de falante da Fase 1. Já na segunda fase do experimento, Lucas apresentou $80 \%$ de acertos e se manteve na Fase 3. Pedro apresentou 100\% de acertos apenas na Fase 3 do estudo, o que representou melhora no desempenho em relação às fases anteriores. As participantes Marina, Cíntia e Daniela obtiveram, no máximo, 60\% de acertos no decorrer de todo o experimento. Para Marina, Lucas e Pedro, é possível observar melhora no desempenho entre os primeiros testes de falante no decorrer do experimento. Os segundos testes de falantes resultaram em $100 \%$ de acertos para praticamente todos os participantes em todas as fases, o que também ocorreu nos testes de ouvinte.
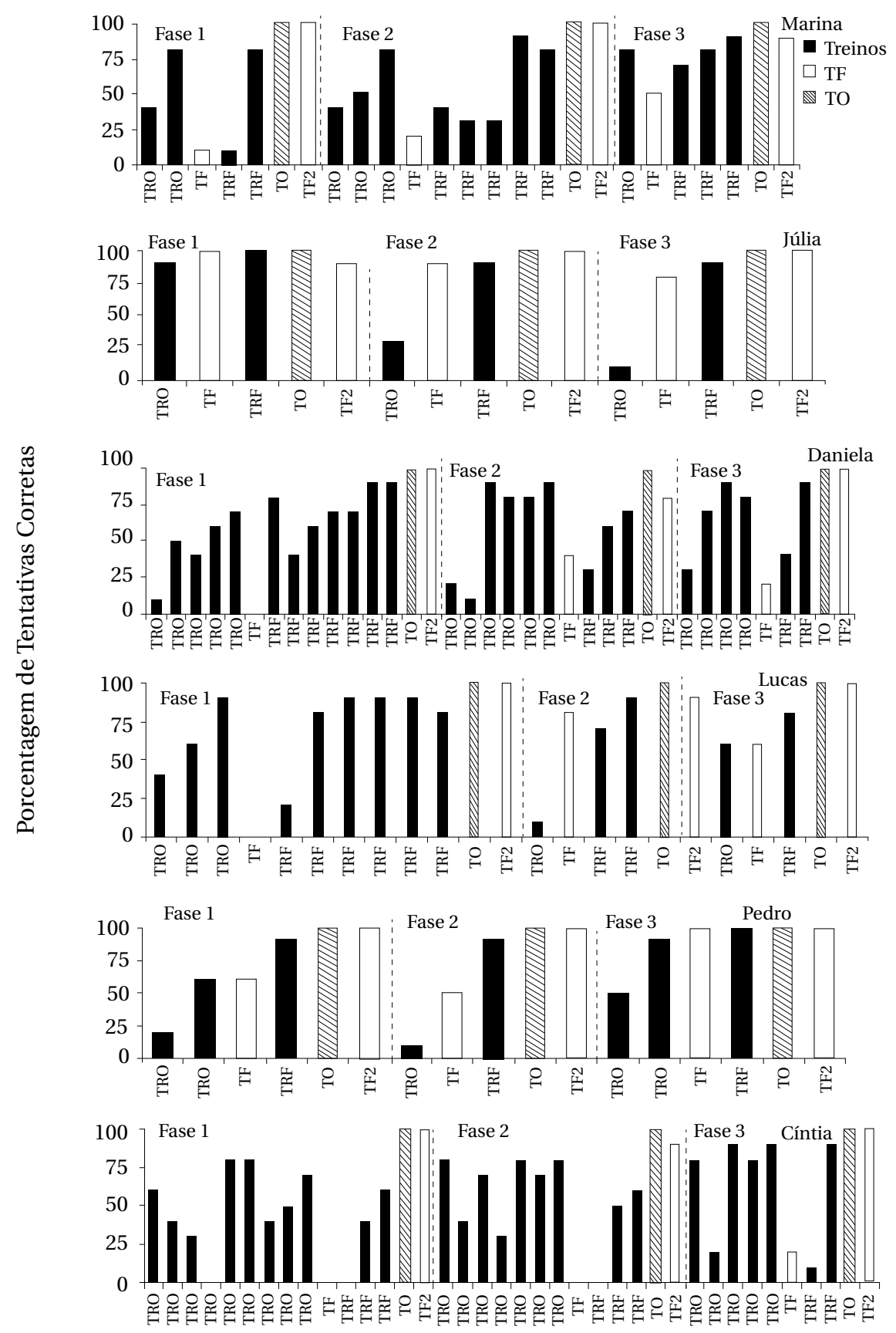

Gráfico 1

Porcentagem de tentativas corretas nos treinos e testes de comportamentos de ouvinte e falante nas três fases do experimento para os participantes expostos à ordem de treino e teste ouvinte-falante. As barras em preto representam os treinos (TRO e TRF); as barras brancas, os testes de falante (TF e TF2); e as barras com listras diagonais, os testes de ouvinte (TO). 
Com relação ao Grupo Falante-Ouvinte, no Gráfico 2, observa-se que para todos eles houve aumento na porcentagem de acertos e diminuição no número de blocos necessários no treino de falante para alcançar o critério estabelecido. Em Bianca, houve menor diferença entre a primeira e a última fase e, para Cristina, maior diferença entre o desempenho no decorrer do experimento. Com relação ao desempenho no primeiro teste de ouvinte de cada fase, apenas Cristina apresentou menos de 100\% de acertos, o que ocorreu na Fase 2. Para os demais participantes, todos acertaram integralmente as tentativas em todos os testes do experimento.
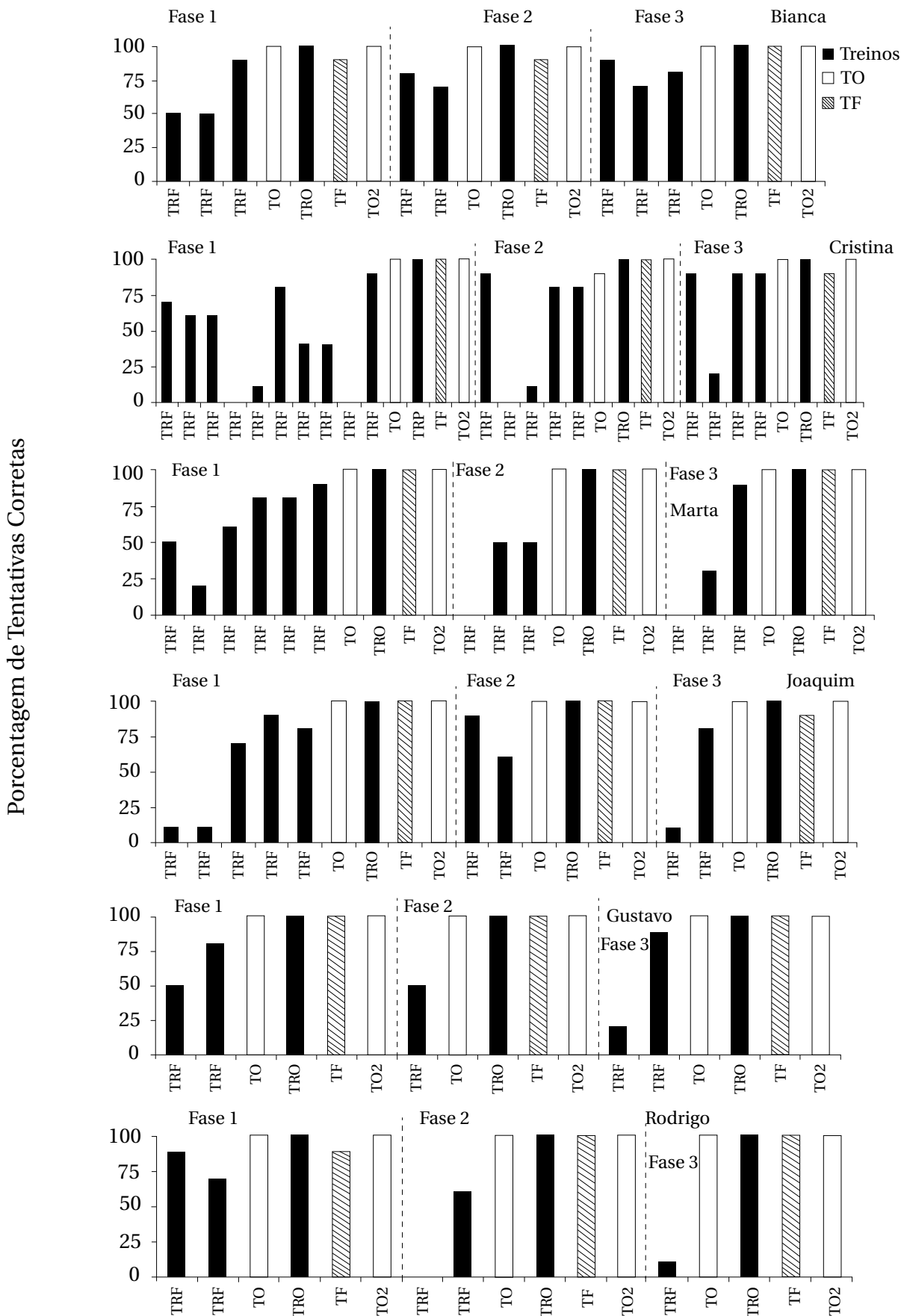

Gráfico 2

Porcentagem de tentativas corretas nos treinos e testes de comportamentos de falante e ouvinte nas três fases do experimento para os participantes expostos à ordem de treino e teste falante-ouvinte. As barras em preto representam os treinos (TRF e TRO); as barras brancas, os testes de ouvinte (TO e TO2); e as barras com listras diagonais, os testes de falante (TF). 


\section{Discussão}

Este trabalho se propôs a investigar a independência funcional entre os comportamentos de ouvinte e falante em adultos e o efeito de treinos sucessivos com a utilização de figuras de paisagem desconhecidas. De maneira geral, os dados apontam que treinos de falante resultam também na emissão não treinada de comportamento de ouvinte, replicando estudos de Bao et al. (2017), Cuvo e Riva (1980), Germano e Medeiros (2020), Lee (1981), Pérez-Gonzáles et al. (2014), e Medeiros et al. (2016), enquanto, nos treinos de ouvinte e testes de falante, há maior variabilidade nos dados (Germano \& Medeiros, 2020; Guess, 1969; Guess \& Baer, 1973; Lee, 198; Viegas \& Medeiros, 2019), sugerindo independência funcional entre esses comportamentos mesmo em participantes adultos.

No que diz respeito à dependência funcional na ordem falante-ouvinte, algumas comparações podem ser feitas com o estudo que serviu de base à elaboração desse procedimento. No Experimento $1 \mathrm{de}$ Medeiros et al. (2016), os autores reportaram que não foi possível observar o efeito dos treinos sucessivos, pois todos os participantes apresentaram desempenho compatível com a dependência funcional nos primeiros testes de ouvinte e pioraram o desempenho na terceira fase experimental. Os autores justificam esses resultados devido à troca de figuras conhecidas para molduras abstratas com caracteres desconhecidos. Essa troca pode ter gerado um controle de estímulo ambíguo, dificultando o desempenho nos testes. Nesta pesquisa, os estímulos utilizados em todas as fases foram figuras de paisagens desconhecidas. Essa mudança metodológica surtiu efeito tendo em vista que os participantes apresentaram desempenho compatível com dependência funcional em todas as fases experimentais. Esse dado dá suporte à hipótese de Medeiros et al. (2016) de que a queda no desempenho na Fase 3 do Experimento 1 pode ser atribuída a um problema de controle de estímulos, corroborando as conclusões dos autores ao replicar os resultados do Experimento 2 sem os caracteres desconhecidos.

Apesar dos dados na ordem falante-ouvinte indicarem a dependência funcional entre esses comportamentos, Medeiros et al. (2016) discutem que esses resultados podem ser encontrados devido ao modo como os comportamentos de falante são treinados. Os autores argumentam que, nos procedimentos de treino de falante do operante mando neste experimento e nos estudos de Germano e Medeiros (2020) e de Medeiros et al. (2016), o experimentador, ao consequenciar o comportamento do participante, emite comportamentos de ouvinte. No caso deste experimento, o experimentador entregava o cartão após o participante emitir a palavra do treino, ou seja, se comportava como ouvinte diante do participante. Dessa forma, o experimentador pode servir de modelo possibilitando a aprendizagem do comportamento de ouvinte por observação (Skinner, 1953).

Ainda com relação aos aspectos dos treinos de falante que favorecem a emissão não treinada dos comportamentos de ouvinte, Sprinkle e Miguel (2012) argumentam que a verbalização da resposta, tanto pelo participante quanto pelo experimentador, pode adquirir função de estímulo discriminativo verbal para o participante se orientar em direção ao estímulo discriminativo não verbal. Esse tipo de comportamento pode ser classificado como comportamento de ouvinte. Nos treinos de falante deste experimento, as topografias de respostas verbais foram ensinadas via prompt ecoico, ou seja, o experimentador emitia a palavra que o participante deveria ecoar diante da sentença e, assim, o cartão com a paisagem correspondente lhe era entregue. Ou seja, diante do nome da figura apresentada pelo experimentador, o participante poderia olhar para a figura que lhe era entregue, o que configuraria um comportamento de ouvinte que poderia guardar relações de similaridade formal e funcional com o comportamento de ouvinte a ser testado.

Pode-se supor, com base nas discussões apresentadas, que os treinos de falante envolvem, ainda que de modo não programado, treinos de comportamentos de ouvinte. Essas características dos treinos de falante ajudariam a compreender a grande incidência da emissão não treinada de comportamentos de ouvinte ao treinar comportamentos de falante. Para verificar essas possibilidades, estudos podem ser conduzidos com o uso de um treino de comportamento de falante que prescinda dos prompts ecoicos e cuja consequenciação da resposta de falante do participante não se constitua num comportamento de ouvinte emitido pelo experimentador.

Quanto aos resultados da ordem ouvinte-falante, observa-se predominância de fases em que os comportamentos foram considerados independentes funcionalmente. Esses dados estão de acordo com os achados na literatura (Germano \& Medeiros, 2020; Guess, 1969; Guess \& Baer, 1973; Lee, 1981; Lemgruber, 2013, Medeiros et al., 2016, Viegas \& Medeiros, 2019). Apesar 
da predominância de independência funcional, para quatro participantes, foi possível observar uma diminuição do número de erros nos testes de falante no decorrer das fases experimentais. Esses resultados apontam para o efeito de treinos sucessivos como em Bernardes e Medeiros (2009), Medeiros et al. (2016), Germano e Medeiros (2020), embora o efeito não tenha sido tão sistemático quanto em Lemgruber (2013), Nuzzolo-Gomes e Greer (2004), Córdova (2008) e para o segundo grupo de Viegas e Medeiros (2019).

$\mathrm{O}$ efeito moderado dos treinos sucessivos neste experimento talvez possa ser explicado ao se considerar o número de estímulos treinados por fase. Em Lemgruber (2013) no segundo grupo de Viegas e Medeiros (2019) e no Experimento 2 de Medeiros et al. (2016) pôde-se observar o efeito dos treinos sucessivos para praticamente todos os participantes que não apresentaram dependência logo na primeira fase. Entre essas pesquisas e o atual experimento, observa-se que há diferença no total de estímulos treinados por fase. Nesta pesquisa, no Experimento 1 de Medeiros et al. (2016) e no estudo de Germano e Medeiros (2020), foram treinados cinco estímulos por fase, os quais reportaram a ausência de efeito de treinos sucessivos ou apenas efeitos moderados desse tipo de treino. Por outro lado, em estudos que utilizaram apenas quatro estímulos por fase, como o segundo experimento de Medeiros et al. (2016), Viegas e Medeiros (2019) e Lemgruber (2013), foi possível observar efeitos mais claros de treinos sucessivos, ou seja, um ganho maior no desempenho ao longo das fases, assim como um número maior de participantes que apresentaram melhora no desempenho no decorrer do experimento. Portanto, uma variável que pode ser crítica quanto ao efeito dos treinos sucessivos é o número de estímulos treinados por fase. Possíveis pesquisas para avaliar esse suposto efeito envolveria a manipulação da quantidade de estímulos treinados e testados por fase.

Algumas análises podem ser feitas quanto aos erros ocorridos nos testes de falante, apesar de eles não terem sido registrados de maneira sistemática. Em Medeiros et al. (2016), pelo fato de os estímulos utilizados serem figuras de objetos do cotidiano, muitos participantes, diante da pergunta do experimentador, emitiram respostas com topografia do nome desses objetos em vez das palavras inventadas para esse experimento. Ou seja, os participantes agiam de acordo com as práticas de reforço da comunidade verbal, mandando os objetos com seus nomes conhecidos (e.g., copo d'água) em vez de emitir as palavras inventadas utilizadas nos treinos de ouvinte (e.g., LITUPE). Para alguns participantes daquele estudo emitirem as palavras inventadas, foi necessário passar por um treino de falante, o qual estabeleceu que, nos testes de falante, as palavras corretas seriam as inventadas, e não as conhecidas dos participantes. Assim, os treinos sucessivos no Experimento 2 de Medeiros et al. (2016) podem ter sido eficazes não por propiciar a dependência funcional entre os comportamentos de ouvinte e falante, e sim por favorecer a emissão das palavras treinadas no lugar de palavras já conhecidas. Como as fases com palavras e objetos conhecidos precederam aquelas com figuras e objetos desconhecidos, os comportamentos requeridos nos testes de falante já poderiam estar estabelecidos, o que resultou na manutenção do desempenho acima do critério de dependência.

Outro padrão de erro observado em Medeiros et al. (2016), Córdova (2008) e Medeiros e Bernardes (2009) foi a emissão do comportamento de apontar como mando genérico em vez da emissão das topografias de respostas requeridas nesses estudos. Ainda que este estudo tenha minimizado a emissão de palavras conhecidas reportada por Medeiros et al. (2016) com o uso de figuras de paisagens desconhecidas, o comportamento de apontar para as figuras, como nos estudos anteriores, foi frequente. Pode-se discutir que os treinos sucessivos tenham sido eficazes em enfraquecer o comportamento de apontar, sendo este considerado como tentativa incorreta nos primeiros testes de falante, o que sugeria independência funcional. Nesse sentido, os treinos sucessivos não propiciam dependência funcional, e sim enfraquecem a emissão de respostas incompatíveis com as contingências programadas (i.e., emitir o nome de figuras conhecidas ou apontar para as figuras). Os resultados de Lucas neste experimento e os de outros dois participantes do Experimento 2 de Medeiros et al. (2016) servem de evidência para essa conclusão, uma vez que apresentaram desempenho compatível com a dependência funcional no primeiro teste de falante da segunda fase do estudo, tendo errado praticamente todas as tentativas do teste de falante da Fase 1.

Egan e Barnes-Holmes (2009, 2010, 2011), em replicações de Nuzzolo-Gomes e Greer (2004) e Lamarre e Holland (1985), discutem os efeitos dos treinos sucessivos, apontando que a independência funcional inicialmente encontrada nesses estudos se deve à forma como a instrução foi dada, configurando os resultados 
desses experimentos em artefatos do método. Ainda que os resultados de Lucas e de dois participantes do Experimento 2 de Medeiros et al. (2016) sirvam de evidência para essa suposição, outros participantes mostraram melhora mais gradual no desempenho ao longo das fases, ou mesmo não apresentaram melhora. Em outras palavras, pode-se supor que os treinos sucessivos tenham efeitos distintos em diferentes participantes, ou mesmo não afetar o comportamento de alguns deles. Nesse sentido, alguns resultados de independência funcional nos primeiros testes de falante não se constituiriam em artefato do método, como sugerem Egan e Barnes-Holmes (2009, 2010, 2011).

Por fim, um último erro ocorrido no teste de falante foi a emissão de palavras com letras ou sílabas trocadas das palavras treinadas. Esse mesmo tipo de erro incidiu em Medeiros et al. (2016) e Germano e Medeiros (2020), em que os participantes emitiram palavras com algumas trocas entre sílabas ou, ainda, com a fusão entre duas palavras no experimento (Skinner, 1957). Viegas e Medeiros (2019) encontraram o mesmo tipo de erro e o discutem com base em controle de estímulos, usando mais especificamente a teoria proposta por Lovaas, Schreibman, Koegel e Rehm (1971) de superseletividade. As palavras utilizadas nesses experimentos foram trissílabas e todas as sílabas diferiram entre as palavras da mesma fase experimental. Desse modo, no treino de ouvinte, bastaria que o comportamento do participante ficasse sob controle de uma propriedade do estímulo (i.e., sílaba) para que ocorresse a emissão de uma resposta considerada correta pelos participantes. Todavia, quando era testado o repertório de falante, caso o comportamento ficasse sob controle apenas de uma propriedade do estímulo, o participante não emitiria a resposta em sua totalidade. Sugere-se, para novas pesquisas, realizar procedimentos em que, no treino de ouvinte, o comportamento de ouvinte fique sob controle de todas as propriedades do estímulo. Uma possibilidade de experimento seria a aplicação de treinos baseados em diferenças críticas, como proposto por Allen e Fuqua (1985).

\section{Considerações finais}

De maneira geral, os resultados dessa pesquisa corroboram a discussão realizada por Skinner (1957), que aponta que os repertórios de ouvinte e falante são independentes funcionalmente, ao menos na direção ouvinte-falante, e que, à medida que os treinos são realizados, observa-se com mais frequência a dependência funcional entre eles. Como apontado no decorrer do trabalho, algumas escolhas metodológicas parecem estar influenciando os resultados obtidos. Dessa maneira, sugere-se que novos estudos façam análises sistemáticas dos possíveis erros nos testes de falante para que esses dados possam contribuir para uma melhor compreensão das variáveis que estão envolvidas no que tange à dependência funcional entre os repertórios de ouvinte e falante.

\section{Referências}

Allen, K. D., \& Fuqua, R. W. (1985). Eliminating selective stimulus control: A comparison of two procedures for teaching mentally retarded children to respond to compound stimuli. Journal of Experimental Child Psychology, 39, 55-71. https://doi.org/10.1016/0022-0965(85)90029-3

Bao, S., Sweatt, K. T., Lechago, S. A., \& Antal, S. (2017). The effects of receptive and expressive instructional sequences of varied conditional discriminations. Journal of Applied Behavior Analysis, 9999, 1-14. https://doi.org/10.1002/jaba.404

Contreras, B. P., Cooper, A. J., \& Kahng, S. (2019). Recent research on the relative efficiency of speaker and listener instruction for children with autism spectrum disorder. Journal of Applied Behavior Analysis, 9999, 1-6. https://doi.org/10.1002/jaba.543

Córdova, L. F. (2008). Efeito de treino sucessivo sobre o comportamento de transposição entre os operantes verbais mando e tato (Tese de doutorado). Universidade de Brasília, Brasília, DF.

Córdova, L. F., Lage, M., \& Ribeiro, A. F. (2007). Relação de independência e dependência funcional entre os operantes verbais mando e tato com a mesma topografia. Revista Brasileira de Análise do Comportamento, 3, 279-298. http://dx.doi.org/10.18542/rebac.v3i2.835

Cuvo, A. J., \& Riva M. T. (1980). Generalization and transfer between comprehension and production: A comparison of retarded and nonretarded persons. Journal of Applied Behavior Analysis, 13(2), 315-331. https://doi.org/10.1901/jaba.1980.13-315 
Egan, C. E., \& Barnes-Holmes, D. (2009). Emergence of tacts following mand training in young children with autism. Journal of applied Behavior Analysis, 42, 691-696. https://doi.org/10.1901/jaba.2009.42-691

Egan, C. E., \& Barnes-Holmes, D. (2010). Establishing mand emergence: the effects of three training procedures and modified antecedent conditions. The Psychological Record, 60, 473-488. https://doi.org/10.1007/BF03395722

Egan, C. E., \& Barnes-Holmes, D. (2011). Examining antecedent control over emergent mands and tacts in young children. The Psychological Record, 61, 127-140. https://doi.org/10.1007/BF03395750

Fiorile, C. A., \& Greer, R. D. (2007). The induction of naming in children with no prior tact responses as a function of multiple exemplar histories of instruction. The Analysis of Verbal Behavior, 23, 71-87. https://doi.org/10.1007/bf03393048

Germano, F. S. S., \& Medeiros. C. A. (2020). Independência funcional entre comportamentos de ouvinte e falante em adultos. Acta Comportamentalia, 28, 391-410.

Gilic, L., \& Greer, R. D. (2011). Establishing naming in typically developing two-year-old children as a function of multiple exemplar speaker and listener experiences. The Analysis of Verbal Behavior, 27, 157-177. https://doi.org/10.1007/bf03393099

Greer, R. D., \& Longano, J. (2010). A Rose by naming: how we may learn how to do it. The Analysis of Verbal Behavior, 26, 73-106. https://doi.org/10.1007/bf03393085

Greer, R. D., \& Speckman, J. (2009). The integration of speaker and listener responses: a theory of verbal development. The Psychological Record, 59, 449-488. https://doi.org/10.1007/BF03395674

Greer, R. D., Stolfi, L., Chavez-Brown, M., \& Rivera-Valdes, C. (2005). The emergence of the listener to speaker component of naming in children as a function of multiple exemplar instruction. The Analysis of Verbal Behavior, 21, 123-134. https://doi.org/10.1007/bf03393014

Guess, D. (1969). A functional analysis of receptive language and productive speech: acquisition of the plural morpheme. Journal of Applied Behavior Analysis, 2, 55-64. https://doi.org/10.1901/jaba.1969.2-55

Guess, D., \& Baer, D. M. (1973). An analysis of individual differences in generalization between receptive and productive language in retarded children. Journal of Applied Behavior Analysis, 6, 311-329. https:// doi.org/10.1901/ jaba.1973.6-311

Hall, G., \& Sundberg, M. L. (1987). Teaching mands by manipulating conditioned establishing operations. The Analysis of Verbal Behavior, 5, 41-53. https:// doi.org/10.1007/BF03392819

Hawkins, E., Gautreaux, G., \& Chiesa, M. (2018). Deconstructing common bidirectional naming: a proposed classification framework. The Analysis of Verbal Behavior, 34, 44-61. https://doi.org/10.1007/s40616-018-0100-7

Horne, P. J., \& Lowe, C. F. (1996). On the origins of naming and other symbolic behavior. Journal of the Experimental Analysis of behavior, 65(1), 185-241. https://doi.org/10.1901/jeab.1996.65-185

LaFrance, D. L. \& Tarbox, J. (2019). The importance of multiple exemplar instruction in the establishment of novel verbal behavior. Journal of Applied Behavior Analysis, 9999, 1-15. https://doi.org/10.1002/jaba.611

Lamarre, J., \& Holland, J. G. (1985). The functional independence of mands and tacts. Journal of the Experimental Analysis of Behavior, 43, 5-19.

Lee,V.L. (1981). Prepositional phrases spoken and heard. JournalofExperimentalAnalysis of Behavior, 35(2), 227-242. https://doi.org/10.1901/jeab.1985.43-5

Lemgruber, L. M. (2013). Efeito de treinos sucessivos na dependência funcional entre comportamentos de ouvinte e falante em crianças (Monografia). Centro Universitário de Brasília, Brasília, DF.

Lovaas, O. I. (2003). Teaching individuals with developmental delays: basic intervention techniques. Austin: Pro-ed.

Lovaas, O. I., Schreibman, L., Koegel, R. L., \& Rehm, R. (1971). Selective responding by autistic children to multiple sensory input. Journal of Abnormal Psychology, 77(3), 211-222. https://doi.org/10.1037/h0031015

Medeiros, C. A., \& Bernardes, M. C. (2009). Estabelecimento de repertório de transposição entre mandos e tatos durante aquisição de nomes de posições. Revista Brasileira de Análise do Comportamento, 5(2), 51-68. http://dx.doi.org/10.18542/rebac.v5i2.930

Medeiros, C. A., Germano, F. S. S., \& Gonçalves, K. L. (2016). Independência funcional em universitários: Funções de ouvinte e falante. Acta Comportamentalia, 24, 419-438. 
Miguel, C. F. (2016). Common and intraverbal bidirectional naming. The Analysis of Verbal Behavior, 32(2), 125-138. https://doi.org/10.1007/s40616-016-0066-2

Nuzzolo-Gomez, R. N., \& Greer, R. D. (2004). Emergence of untaught mands or tacts of novel adjective-object pairs as a function of instructional history. The Analysis of Verbal Behavior, 20, 63-76. https://doi.org/10.1007/BF03392995

Pérez-Gonzáles, L. A., Cereijo-Blanco, N., \& Carnerero, J. J. (2014). Emerging tacts and selections from previous learned skills: a comparison between two types of naming. The Analysis of Verbal Behavior, 30(2), 184-192. https://doi.org/10.1007/s40616-014-0011-1

Petursdottir, A. I., \& Carr, J. E. (2011). A review of recommendations for sequencing receptive and expressive language instruction. Journal of Applied Behavior Analysis, 44, 859-876. https://doi.org/10.1901/jaba.2011.44-859

Skinner B. F. (1953). Science and human behavior. New York: Macmillan.

Skinner, B. F. (1957). Verbal behavior. New York: Appleton-Century-Crofts.

Skinner, B. F. (1991). Questões recentes na análise comportamental (A. L. Neri, Trad.). Campinas, SP: Papirus. (Trabalho original publicado em 1989)

Sprinkle, E. C., Miguel, C. F. (2012). The effects of listener and speaker training on emergent relations in children with autism. Analysis Verbal Behavavior, 28, 111-117. https://doi.org/10.1007/BF03393111

Viegas, E. W., \& Medeiros, C. A. (2019). Dependência funcional das funções de ouvinte e falante: efeito da quantidade de treino. Acta Comportamentalia, 27(2), 127-143.

Wynn, J. W., \& Smith, T. (2003). Generalization between receptive and expressive language in young children with autism. Behavioral Interventions, 18, 245-266. https://doi.org/10.1002/bin.142

\section{Sarah Moraes Simões}

Instituto Brasiliense de Análise do Comportamento (IBAC), Brasília - DF. Brasil.

E-mail: sarahsimoespsi@gmail.com

(1) https://orcid.org/0000-0001-8654-8162

\section{Carlos Augusto Medeiros}

Professor titular. Mestrado em Psicologia pelo Instituto CEUB de Pesquisa e Desenvolvimento. Centro Universitário de Brasília (UniCEUB), Brasília - DF. Brasil.

E-mail: medeiros.c.a@gmail.com

(1) https://orcid.org/0000-0003-0661-9739

\section{Eduardo Walcacer Viegas}

Egresso do Programa de Mestrado em Psicologia do Centro Universitário de Brasília (UniCEUB), Brasília - DF. Brasil E-mail: edutsviegas@gmail.com

(D) https://orcid.org/0000-0003-2385-3391

Endereço para envio de correspondência:

Secretaria de Pós-Graduação Stricto Sensu. Campus do UniCEUB, bloco 3, SEPN 707/907, Asa Norte. CEP 70790-075. Brasília-DF. Brasil.

Recebido 30/05/2018

Aceito 30/03/2020

Received 30/05/2018

Approved 30/03/2020

Recibido 30/05/2018

Aceptado 30/03/2020 
Como citar: Simões, S. M., Medeiros, C. A., \&Viegas, E. W. (2020). Independência Funcional entre Comportamentos de Falante e Ouvinte Relativos a Paisagens Desconhecidas. Psicologia: Ciência e Profissão, 40, 1-15. https://doi.org/10.1590/1982-3703003201570

How to cite: Simões, S. M., Medeiros, C. A., \& Viegas, E. W. (2020). Functional Independence Between SpeakerListener Behaviors Related to Unknown Landscapes. Psicologia: Ciência e Profissão, 40, 1-15.

https://doi.org/10.1590/1982-3703003201570

Cómo citar: Simões, S. M., Medeiros, C. A., \& Viegas, E. W. (2020). Independencia Funcional entre Comportamientos de Hablante y Oyente Relacionados a Paisajes Desconocidos. Psicologia: Ciência e Profissão, 40, 1-15. https://doi.org/10.1590/1982-3703003201570 